fournal of Medical Genetics (1974). 11, 280.

\title{
Familial translocation $15 / 22$. A possible cause for abortions in female carriers
}

\author{
K. FRIED, J. BUKOVSKY, M. ROSENBLATT, and G. MUNDEL \\ From the Departments of Genetics, Gynaecology and Obstetrics, and Paediatrics, Asaf Harofe Government Hospital, \\ Tel-Aviv University Medical School, Zerifin, Israel
}

\begin{abstract}
Summary. A familial Robertsonian translocation $15 / 22$ was ascertained through a female carrier whose four pregnancies ended in missed abortions. Eleven $15 / 22$ translocation carriers were detected in three generations among 23 family members investigated. The four proven female carriers, apart from the proposita, have miscarried seven out of 14 pregnancies. The kindred suggests that the 15/22 translocation in female carriers may cause an increased risk for miscarriage.
\end{abstract}

In a study of 5049 consecutive newborn children, Friedrich and Nielsen (1973) found one boy who had a translocation $45, \mathrm{XY}, \mathrm{t}(15 \mathrm{q} 22 \mathrm{q})$, which was inherited from the mother. He was physically normally developed; his family has been described by Friedrich, Nielsen, and Sehested (1972). To the best of our knowledge this was the only reported family with a $D / G$ translocation positively identified as a $15 / 22$ translocation by the banding technique.

\section{Methods}

Chromosome preparations were made from cultures of peripheral blood by the method of Moorhead et al (1960). Colchicine was added to the cultures 2 hours before harvesting. The hypotonic solution used was $\mathbf{0 . 0 7 5}$ M KC1 for 10 minutes. The fixative, methanol:acetic

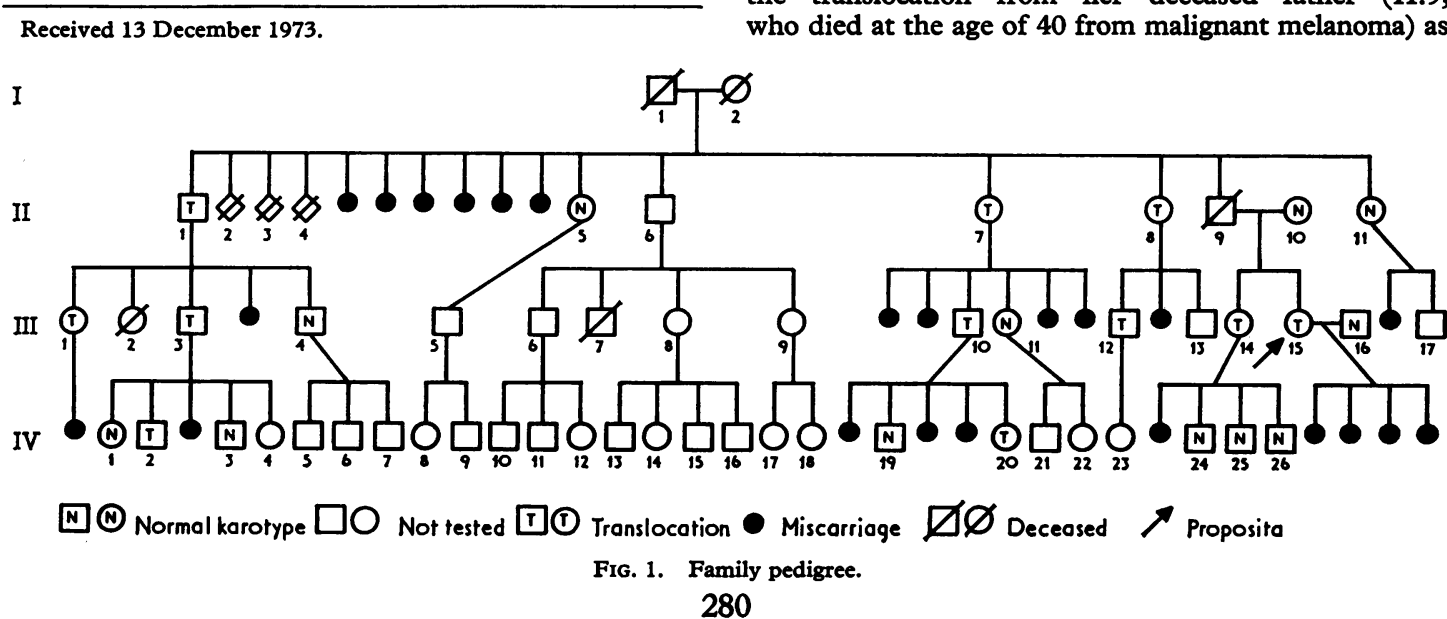

acid (3:1), was added and slides were prepared by the air dry technique. The ASG (acetic/saline/Giemsa) technique was used for banding chromosomes. The slides were incubated for 1 hour at $60^{\circ} \mathrm{C}$ in $2 \times \mathrm{SSC}$ ( $0.3 \mathrm{M}$ sodium chloride plus $0.03 \mathrm{M}$ tri-sodium citrate) after which they were rinsed briefly with de-ionized water and stained in Giemsa for 30 minutes (Gurr's Giemsa R.66, $2 \mathrm{ml}$ to $50 \mathrm{ml}$ of buffer at pH 6.8 made with Gurr's buffer tablets). Finally they were rinsed again briefly in de-ionized water and dried by blotting.

\section{Family report}

The family was ascertained because the proposita had four pregnancies all of which ended in missed abortions. The proposita (III.15, Fig. 1) was found to be a translocation carrier $45, \mathrm{XX}, \mathrm{t}(15 \mathrm{q} 22 \mathrm{q})$, her husband had a normal karyotype. She must have inherited the translocation from her deceased father (II.9, who died at the age of $\mathbf{4 0}$ from malignant melanoma) as 


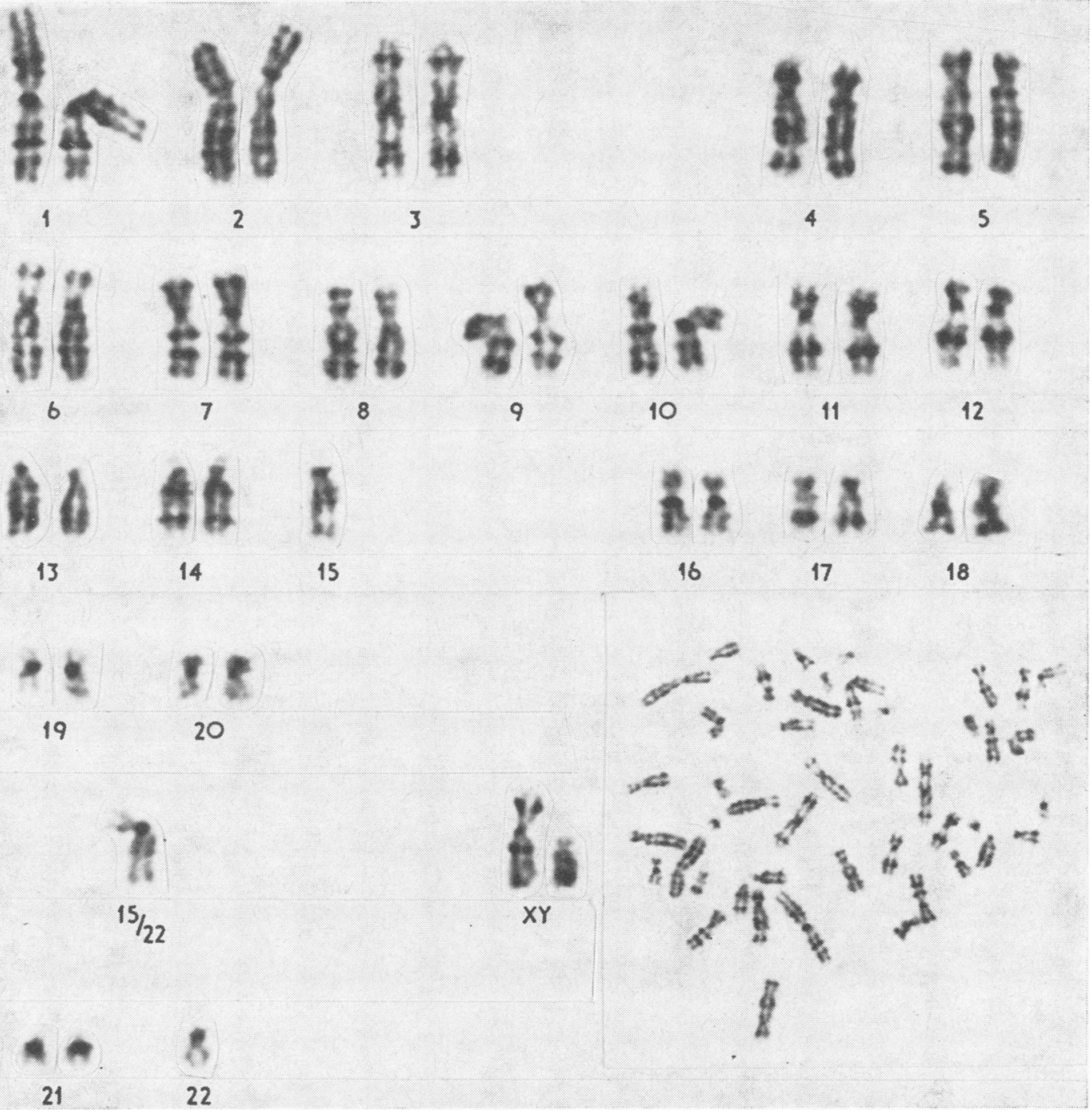

FIG. 2. Karyotype of IV.2, one of the $15 / 22$ Robertsonian translocation carriers. (ASG staining.)

some of his sibs were found to be carriers of the same translocation. The grandparents (I.1 and I.2) were both deceased. The grandfather and grandmother died at the age of 80 and 73, respectively. All the available and cooperative sibs of translocation carriers and their offspring were karyotyped (Figs. 1 and 2). There were 11 karyotyped translocation carriers in the kindred; five of them were males. All the translocation carriers were physically and mentally normal, some being university graduates. Relatives with normal karyotypes were also normal except IV.26, a nephew of the proposita who was severely mentally retarded with some areas of depigmentation of the skin. All individuals who were karyotyped were interviewed and complete conceptual histories were obtained before karyotyping. Apart from the proposita, the four other proven adult female carriers (II.7, II.8, III.1, and III.14) have miscarried seven out of 14 pregnancies. The majority of miscarriages were in the first trimester but some were in the second and one early in the third trimester. The wives of the four married proven male translocation carriers (II.1, III.3, III.10, and III.12) had 16 pregnancies, five of which ended in spontaneous abortions. One of their offspring (III.2) died as a newborn because of a spinal defect. I.2 is reported to have had six spontaneous abortions and three children who died (the order of the pregnancies 
of I.2 is not known). Either I.2 or I.1 is presumed to have been a translocation carrier as they had several translocation carrier children.

\section{Discussion}

The family studied by Friedrich et al (1972) did not show any abortions in female $15 / 22$ translocation carriers, but the family was very small and the translocation carriers had only very few pregnancies. Infertility has been recorded in balanced $t(D q D q)$ heterozygotes (Walker and Harris, 1962/1963; Kjessler, 1964; Yunis et al, 1964; Wilson, 1971). Sparkes and de Chieri (1970) suggested that the carrier of a chromosome translocation might be at increased risk for having recurrent abortions. At least one female 14/21 translocation carrier was ascertained because of multiple abortions (Cohen, 1971).

The finding of seven miscarriages among 14 pregnancies of four 15/22 translocation heterozygotes suggests a causal effect. The proposita, who was ascertained because of four missed abortions, was excluded from the calculation, but this may be an overcorrection for ascertainment bias.

The finding of five miscarriages among 16 pregnancies of the wives of four male $15 / 22$ translocation heterozygotes may also be indicative of a trend but the data from this family are too few to reach any conclusions about fetal wastage of male translocation carriers or segregation ratios. Reports of more families with this type of translocation may provide such data. It seems that karyotyping the abortions of the translocation carriers may provide important information about the cause of the abortions.

\section{Addendum}

Two further balanced carriers of $t(15 q 22 q)$ were reported by Jacobs et al (1974). One of them, who was ascertained through a survey of prisoners, has inherited the translocation from his mother. The other, ascertained through a survey of patients in a mental subnormality hospital, was a new mutant.

\section{REFBRENCES}

Cohen, M. M. (1971). The chromosomal constitution of 165 human translocations involving D group chromosomes identified by autoradiography. Annales de Génétique, 14, 87-96.

Friedrich, U. and Nielsen, J. (1973). Chromosome studies in 5049 consecutive newborn children. Clinical Genetics, 4, 333-343.

Friedrich, U., Nielsen, J., and Sehested, J. (1972). A family with 15/22 translocation. Hereditas, Genetiskt Arkiv. 72, 172-174.

Jacobs, P. A., Buckton, K. E., Cunningham, C., and Newton, M (1974). An analysis of the break points of structural rearrangements in man. Fournal of Medical Genetics, 11, 50-64.

Kjessler, B. (1964). Meiosis in a man with a $D / D$ translocation and clinical sterility. Lancet, 1, 1421-1423.

Moorhead, P. S., Nowell, P. C., Mellman, W. J., Batipps, D. M., and Hungerford, D. A. (1960). Chromosome preparations of leukocytes cultured from human peripheral blood. Experimental Cell Research, 20, 613-616.

Sparkes, R. S. and De Chieri, P. R. (1970). Inherited 13/14 chromosome translocation as a cause of human fetal wastage. Obstetrics and Gynecology, 35, 601-607.

Walker, S. and Harris, R. (1962/1963). Familial transmission of a translocation between two chromosomes of 13-15 group. (Denver classification.) Annals of Human Genetics, 26, 151-162.

Wilson, J. A. (1971). Fertility in balanced heterozygotes for a familial centric fusion translocation, $t(D q D q)$. Fournal of Medical Genetics, 8, 175-178.

Yunis, J. J., Alter, M., Hook, E. B., and Mayer, M. (1964). Familial D-D translocation. Report of a pedigree and DNA replication analysis. New England fournal of Medicine, 271, 1133-1137. 